\title{
Prognostic Significance of Preoperative Fibrinogen-to- Prealbumin Ratio in Patients with Stage I-III Colorectal Cancer Undergoing Surgical Resection: A Retrospective Cohort Study
}

\author{
Hailun Xie, ${ }^{1}$ Shizhen Huang, ${ }^{1}$ Guanghui Yuan, ${ }^{1}$ Shuangyi Tang, ${ }^{2}$ and Jialiang Gan $\mathbb{D}^{1}$ \\ ${ }^{1}$ Department of Colorectal and Anal Surgery, The First Affiliated Hospital, Guangxi Medical University, Nanning 530021, China \\ ${ }^{2}$ Department of Pharmacy, The First Affiliated Hospital, Guangxi Medical University, Nanning 530021, China \\ Correspondence should be addressed to Jialiang Gan; gj15172@163.com
}

Received 7 August 2020; Revised 11 November 2020; Accepted 4 January 2021; Published 13 January 2021

Academic Editor: Bing Wang

Copyright (C) 2021 Hailun Xie et al. This is an open access article distributed under the Creative Commons Attribution License, which permits unrestricted use, distribution, and reproduction in any medium, provided the original work is properly cited.

\begin{abstract}
Background. The objective of this study was to explore the role of preoperative fibrinogen-to-prealbumin ratio (FPR) in evaluating the prognosis of patients with stage I-III colorectal cancer (CRC). Methods. This retrospective study enrolled 584 stage I-III CRC patients undergoing surgical resection. Logistic regression analysis was used to explore the correlation between FPR and postoperative complications. The Kaplan-Meier curve and Cox proportional hazards model were used to identify the prognostic factors. The nomograms were constructed based on the prognostic factors. The concordance index and calibration curve were used to determine the accuracy of the nomograms. Time-dependent receiver operating characteristic was used to compare the predictive prognostic efficacy of nomograms and TNM stage. Results. FPR was determined to be an independent factor affecting postoperative complications. Patients with a low-FPR had a significantly better prognosis than those with a high-FPR (diseasefree survival, $p=0.028$; overall survival, $p=0.027$ ), especially patients with stage I CRC (disease-free survival, $p=0.015$; overall survival, $p=0.017$ ). The Cox proportional hazards model identified FPR as an independent poor prognostic factor of diseasefree survival (hazard ratio $(\mathrm{HR})=1.459,95 \%$ confidence interval $(\mathrm{CI})=1.074-1.954, p=0.011$ ) and overall survival $(\mathrm{HR}=1.405,95 \% \mathrm{CI}=1.034-1.909, p=0.030)$. The prognostic nomograms had good accuracy and were superior to the traditional TNM stage. Conclusions. FPR is a potential indicator for predicting short- and long-term prognosis of stage I-III CRC patients undergoing surgical resection.
\end{abstract}

\section{Introduction}

Colorectal cancer (CRC) is currently the third most common malignancy in the world, with 1.8 million new cases diagnosed every year. It is also the second leading cause of cancer-related deaths worldwide, accounting for approximately one-tenth of the total number of deaths [1]. Surgical resection of the primary tumor and subsequent adjuvant chemotherapy are still the main treatment strategies for CRC patients. With the advancement of surgical and adjuvant therapy techniques, the five-year overall survival (OS) in CRC patients has improved. However, a large proportion of patients undergoing primary tumor resection still die from tumor recurrence or metastasis [2, 3]. Hence, novel, effective, and economical prognostic biomarkers are urgently needed for prognosis evaluation and formulating individualized treatment strategies in CRC patients.

Inflammation and nutritional status are well known to play important roles in cancer patient survival. Tumorpromoting inflammation is a hallmark of cancer. An increasing number of studies have demonstrated that inflammation is closely related to carcinogenesis and progression of CRC $[4,5]$ and is one of the most critical reasons for the occurrence and metastasis of CRC [6]. In addition, Friis et al. have found that long-term use of low-dose non-steroidal antiinflammatory drugs can reduce susceptibility to CRC [7]. Nutritional status is another important factor affecting the prognosis of CRC patients. Malnutrition can lead to 
TABLE 1: The correlations between the FPR and clinicopathological factors.

\begin{tabular}{|c|c|c|c|c|c|}
\hline \multirow{2}{*}{ Variable } & \multirow{2}{*}{ Case (584) } & \multicolumn{2}{|c|}{ FPR } & \multirow{2}{*}{$\chi^{2} / t$} & \multirow{2}{*}{$p$} \\
\hline & & Low (357) & High (227) & & \\
\hline Sex & & & & 0.796 & 0.372 \\
\hline Male & $363(62.2 \%)$ & $227(63.6 \%)$ & $136(59.9 \%)$ & & \\
\hline Female & $221(37.8 \%)$ & $130(36.4 \%)$ & $91(40.1 \%)$ & & \\
\hline Age (years) & $57.57 \pm 13.41$ & $56.31 \pm 12.76$ & $59.56 \pm 14.16$ & 2.877 & 0.004 \\
\hline BMI & $22.11 \pm 3.42$ & $22.49 \pm 3.35$ & $21.51 \pm 3.44$ & -3.385 & 0.001 \\
\hline Diabetes mellitus & & & & 3.513 & 0.061 \\
\hline Negative & $553(94.7 \%)$ & $343(96.1 \%)$ & $210(92.5 \%)$ & & \\
\hline Positive & $31(5.3 \%)$ & $14(3.9 \%)$ & $17(7.5 \%)$ & & \\
\hline Hypertension & & & & 2.114 & 0.146 \\
\hline Negative & $492(84.2 \%)$ & $307(86.0 \%)$ & $185(81.5 \%)$ & & \\
\hline Positive & $92(15.8 \%)$ & $50(14.0 \%)$ & $42(18.5 \%)$ & & \\
\hline Cardiovascular disease & & & & 0.096 & 0.757 \\
\hline Negative & $570(97.6 \%)$ & $349(97.8 \%)$ & $221(97.4 \%)$ & & \\
\hline Positive & $14(2.4 \%)$ & $8(2.2 \%)$ & $6(2.6 \%)$ & & \\
\hline Depth of invasion & & & & 1.178 & 0.278 \\
\hline $\mathrm{T} 1-2$ & $164(28.1 \%)$ & $106(29.7 \%)$ & $58(25.6 \%)$ & & \\
\hline T3-4 & $420(71.9 \%)$ & $251(70.3 \%)$ & $169(74.4 \%)$ & & \\
\hline Lymph node status & & & & 4.197 & 0.123 \\
\hline N0 & $341(58.4 \%)$ & $208(58.3 \%)$ & $133(58.6 \%)$ & & \\
\hline N1 & $162(27.7 \%)$ & $92(25.8 \%)$ & $70(30.8 \%)$ & & \\
\hline N2 & $81(13.9 \%)$ & $57(16.0 \%)$ & $24(10.6 \%)$ & & \\
\hline Tumor stage & & & & 0.213 & 0.899 \\
\hline I & $125(21.4 \%)$ & $78(21.8 \%)$ & $47(20.7 \%)$ & & \\
\hline II & $215(36.8 \%)$ & $129(36.1 \%)$ & $86(37.9 \%)$ & & \\
\hline III & $244(41.8 \%)$ & $150(42.0 \%)$ & $94(41.4 \%)$ & & \\
\hline Tumor location & & & & 21.511 & $<0.001$ \\
\hline Rectal & $317(54.3 \%)$ & $221(61.9 \%)$ & $96(42.3 \%)$ & & \\
\hline Colon & $267(45.7 \%)$ & $136(38.1 \%)$ & $131(57.7 \%)$ & & \\
\hline Perineural invasion & & & & 0.125 & 0.724 \\
\hline Negative & $533(91.3 \%)$ & $327(91.6 \%)$ & $206(90.7 \%)$ & & \\
\hline Positive & $51(8.7 \%)$ & $30(8.4 \%)$ & $21(9.3 \%)$ & & \\
\hline \multicolumn{6}{|l|}{ Vascular invasion } \\
\hline Negative & $501(85.8 \%)$ & $316(88.5 \%)$ & $185(81.5 \%)$ & 5.605 & 0.018 \\
\hline Positive & $83(14.2 \%)$ & $41(11.5 \%)$ & $42(18.5 \%)$ & & \\
\hline Pathological type & & & & 2.139 & 0.343 \\
\hline Protrude type & $117(20.0 \%)$ & $68(19.0 \%)$ & $49(21.6 \%)$ & & \\
\hline Infiltrating type & $69(11.8 \%)$ & $38(10.6 \%)$ & $31(13.7 \%)$ & & \\
\hline Ulcerative type & $398(68.2 \%)$ & $251(70.3 \%)$ & $147(64.8 \%)$ & & \\
\hline Histological grade & & & & 0.834 & 0.361 \\
\hline Low & $61(10.4 \%)$ & $34(9.5 \%)$ & $27(11.9 \%)$ & & \\
\hline High & $523(89.6 \%)$ & $323(90.5 \%)$ & $200(88.1 \%)$ & & \\
\hline Tumor size & $4.93 \pm 2.01$ & $4.36 \pm 1.60$ & $5.83 \pm 2.25$ & 8.591 & $<0.001$ \\
\hline ASA classification & & & & 0.291 & 0.590 \\
\hline I-II & $322(55.1 \%)$ & $200(56.0 \%)$ & $122(53.7 \%)$ & & \\
\hline III-IV & $262(44.9 \%)$ & $157(44.0 \%)$ & $105(46.3 \%)$ & & \\
\hline
\end{tabular}


TABle 1: Continued.

\begin{tabular}{|c|c|c|c|c|c|}
\hline \multirow{2}{*}{ Variable } & \multirow[b]{2}{*}{ Case (584) } & \multicolumn{2}{|c|}{ FPR } & \multirow{2}{*}{$\chi^{2} / t$} & \multirow[b]{2}{*}{$p$} \\
\hline & & Low (357) & High (227) & & \\
\hline CEA & & & & 6.451 & 0.011 \\
\hline Normal & $369(63.2 \%)$ & $240(67.2 \%)$ & $129(56.8 \%)$ & & \\
\hline High & $215(36.8 \%)$ & $117(32.8 \%)$ & $98(43.2 \%)$ & & \\
\hline Length of hospitalization & $14.44 \pm 6.98$ & $14.15 \pm 6.33$ & $14.89 \pm 7.88$ & 1.248 & 0.213 \\
\hline Albumin & $36.90 \pm 4.00$ & $38.33 \pm 3.13$ & $34.64 \pm 4.18$ & -12.164 & $<0.001$ \\
\hline Prealbumin & $203.88 \pm 60.65$ & $235.08 \pm 47.42$ & $154.80 \pm 44.60$ & -20.686 & $<0.001$ \\
\hline Fibrinogen & $4.29 \pm 1.03$ & $3.82 \pm 0.72$ & $5.04 \pm 0.98$ & 17.364 & $<0.001$ \\
\hline PNI & $46.26 \pm 6.38$ & $48.12 \pm 4.85$ & $43.33 \pm 7.34$ & 8.689 & $<0.001$ \\
\hline NLR & $2.72 \pm 2.36$ & $2.26 \pm 1.70$ & $3.44 \pm 3.00$ & 5.389 & $<0.001$ \\
\hline LMR & $3.85 \pm 1.93$ & $4.20 \pm 1.70$ & $3.30 \pm 2.13$ & -6.776 & $<0.001$ \\
\hline PLR & $158.76 \pm 87.24$ & $138.71 \pm 71.83$ & $190.29 \pm 99.35$ & 30.271 & $<0.001$ \\
\hline Postoperative complications $(\mathrm{CD} \geq 2)$ & $69(11.8 \%)$ & $32(9.0 \%)$ & $37(16.3 \%)$ & 7.167 & 0.007 \\
\hline
\end{tabular}

undesirable clinical consequences, such as reduced body immunity, decreased therapeutic efficacy, and postoperative complications [8].

It has been recently reported that fibrinogen-toprealbumin ratio (FPR) can be utilized to predict the prognosis of various malignancies, such as hepatocellular carcinoma, gastric cancer, and esophageal cancer [9-13]. Fibrinogen can promote the synthesis of proinflammatory cytokines and fibroblast growth factors, induce malignant tumor cell proliferation, promote tumor angiogenesis, and participate in cell responses related to tumor cell adhesion and migration [14, 15]. Several studies have demonstrated that preoperative plasma fibrinogen levels can independently predict the prognosis of various malignancies [16-18] including CRC. Prealbumin is an essential nutritional indicator with a short half-life that is considered to be more sensitive than albumin in reflecting protein energy status changes. It is also an effective biomarker that timely reflects inflammatory stress and tumor progression [19,20]. Many studies have reported that low preoperative prealbumin level is associated with poor prognosis of various malignancies [21, 22]. Thus, FPR combined with fibrinogen and prealbumin can more reliably reflect the inflammatory and nutritional status of the host and is a potentially promising prognostic indicator.

At present, few researchers have reported the prognostic value of FPR in CRC patients, and no studies have explored the correlation between FPR and postoperative complications in CRC patients. Therefore, the present study retrospectively analyzed patient data from our center to explore the value of FPR in short- and long-term outcomes of stage I-III CRC patients undergoing surgical resection.

\section{Materials and Methods}

2.1. Patient Selection. A total of 584 patients with pathologically confirmed stage I-III CRC who underwent surgical resection at the First Affiliated Hospital of Guangxi Medical University between January 2012 and December 2014 were retrospectively analyzed. All enrolled patients underwent radical resection without preoperative neoadjuvant chemoradiotherapy. The exclusion criteria included patients presenting with systemic infection, hematological diseases, and additional malignancies, as well as individuals with incomplete preoperative serological data or those lost during postoperative follow-up. The research was approved by the institutional Ethics Committee of our center. Written informed consent was obtained from each patient.

2.2. Clinical Parameters and Laboratory Results. All enrolled patients were measured for serum neutrophil count, monocyte count, lymphocyte count, platelet count, fibrinogen, albumin, prealbumin, and carcinoembryonic antigen (CEA) levels within one week before surgery. Clinical data were collected using the hospital information system and included time of admission, length of hospitalization, gender, age, body mass index (BMI), preoperative comorbidities (diabetes, hypertension, and cardiovascular disease), postoperative chemotherapy, tumor location, type of surgery, depth of invasion, perineural invasion, vascular invasion, pathological type, histological grade, tumor size, blood loss, and operation duration. The comorbidities were classified according to the American Society of Anesthesiologists (ASA) classification. Tumor stages were classified according to the 8th tumornode-metastasis (TNM) classification system of CRC. Prognostic study indicators were as follows: FPR = fibrinogen-to-prealbumin ratio, NLR = neutrophil-to-lymphocyte ratio, $\mathrm{LMR}=$ lymphocyte-to-monocyte ratio, PLR = platelet-to-lymphocyte ratio, and prognostic nutritional index $(\mathrm{PNI})=\operatorname{albumin}(\mathrm{g} / \mathrm{L})+5 \times$ lymphocyte $\left(10^{9} / \mathrm{L}\right)$.

2.3. Survival and Follow-Up. Follow-up data were collected by telephone interviews and retrieving outpatient and inpatient medical records. All included patients were regularly followed up every 3-6 months during the first two years and then every six months until September 1, 2019. The follow-up data consisted of medical history, physical examination, tumor marker detection, tumor assessment imaging, 
TABLE 2: The correlations between the postoperative complications and various clinicopathological factors.

\begin{tabular}{|c|c|c|c|c|c|}
\hline \multirow{3}{*}{ Variable } & \multicolumn{5}{|c|}{ Postoperative complication } \\
\hline & \multicolumn{2}{|c|}{ Univariate } & \multicolumn{3}{|c|}{ Multivariate } \\
\hline & HR (95\% CI) & $p$ value & $\operatorname{HR}(95 \% \mathrm{CI})$ & $p$ value & $\beta$ \\
\hline Gender (female) & $0.801(0.471-1.361)$ & 0.412 & & & \\
\hline Age (years) $(\geq 60)$ & $1.675(1.002-2.799)$ & 0.049 & $1.311(0.756-2.273)$ & 0.335 & 0.271 \\
\hline BMI & & 0.876 & & & \\
\hline Low & 1 & & & & \\
\hline Normal & $1.071(0.514-2.232)$ & & & & \\
\hline High & $0.916(0.400-2.100)$ & & & & \\
\hline Diabetes mellitus (positive) & $1.867(0.737-4.726)$ & 0.188 & & & \\
\hline Hypertension (positive) & $2.103(1.165-3.798)$ & 0.014 & $1.843(0.984-3.450)$ & 0.056 & 0.611 \\
\hline Cardiovascular disease (positive) & $1.251(0.274-5.712)$ & 0.772 & & & \\
\hline Depth of invasion (T3-4) & $1.031(0.588-1.809)$ & 0.914 & & & \\
\hline Lymph node status & & 0.987 & & & \\
\hline No & 1.000 & & & & \\
\hline N1 & $1.000(0.559-1.788)$ & & & & \\
\hline $\mathrm{N} 2$ & $1.060(0.506-2.221)$ & & & & \\
\hline Tumor location (colon) & $0.844(0.507-1.403)$ & 0.513 & & & \\
\hline Perineural invasion (positive) & $1.209(0.522-2.801)$ & 0.659 & & & \\
\hline Vascular invasion (positive) & $1.476(0.767-2.838)$ & 0.243 & & & \\
\hline Pathological type & & 0.693 & & & \\
\hline Protrude type & 1.000 & & & & \\
\hline Infiltrating type & $0.701(0.256-1.917)$ & & & & \\
\hline Ulcerative type & $1.033(0.548-1.946)$ & & & & \\
\hline Histological grade (low) & $1.764(0.869-3.579)$ & 0.116 & & & \\
\hline Tumor size $(\geq 5 \mathrm{~cm})$ & $1.614(0.968-2.689)$ & 0.066 & & & \\
\hline CEA (high) & $0.972(0.576-1.639)$ & 0.915 & & & \\
\hline ASA grade (III-IV) & $1.072(0.647-1.774)$ & 0.788 & & & \\
\hline Type of surgery (open) & $1.041(0.630-1.722)$ & 0.874 & & & \\
\hline Albumin (low) & $1.278(0.743-2.196)$ & 0.375 & & & \\
\hline Prealbumin (low) & $1.200(0.704-2.044)$ & 0.502 & & & \\
\hline Fibrinogen (high) & $1.233(0.883-1.720)$ & 0.219 & & & \\
\hline FPR (high) & $1.978(1.193-3.280)$ & 0.008 & $1.785(1.062-2.999)$ & 0.029 & 0.579 \\
\hline PNI (low) & $1.540(0.924-2.566)$ & 0.097 & & & \\
\hline NLR (high) & $1.104(0.667-1.825)$ & 0.701 & & & \\
\hline LMR (high) & $0.649(0.390-1.082)$ & 0.097 & & & \\
\hline PLR (high) & $1.440(0.866-2.393)$ & 0.160 & & & \\
\hline Blood loss $(\geq 100 \mathrm{~mL})$ & $2.034(1.204-3.438)$ & 0.008 & $1.956(1.151-3.326)$ & 0.013 & 0.671 \\
\hline Operation time ( $\geq 209 \mathrm{~min})$ & $1.540(0.924-2.566)$ & 0.097 & & & \\
\hline
\end{tabular}

and colonoscopy. The clinical outcomes included postoperative complications, disease-free survival (DFS), and OS. Postoperative complications were defined using Clavien-Dindo (CD) classification of grade II or higher [23]. DFS was calculated from the date of operation until recurrence, metastasis, death, or the last follow-up. OS was calculated from the date of operation until death or last follow-up.

2.4. Statistical Analysis. The optimal threshold value for FPR was determined using X-tile software version 3.6.1 (Yale University School of Medicine, New Haven, CT, USA). The differences between groups were compared using a chi-square test, Fisher's exact test, or Student's $\boldsymbol{t}$-test as appropriate. Univariate/multivariate logistic regression analysis was performed to investigate the relationship between FPR and postoperative complications. The Kaplan-Meier curve and logrank test were used to assess the differences in survival rate. Survival analysis was assessed using the Cox proportional hazards model to determine potential prognostic factors. Factors with $\boldsymbol{p}$ values $<0.05$ in univariate analysis were used for multivariate analysis. R software (Version 3.5.3; https:// www.R-project.org) was used to establish the survival nomograms. Then, the performance of prognostic nomograms was evaluated using the concordance index ( $\boldsymbol{C}$-index) and 

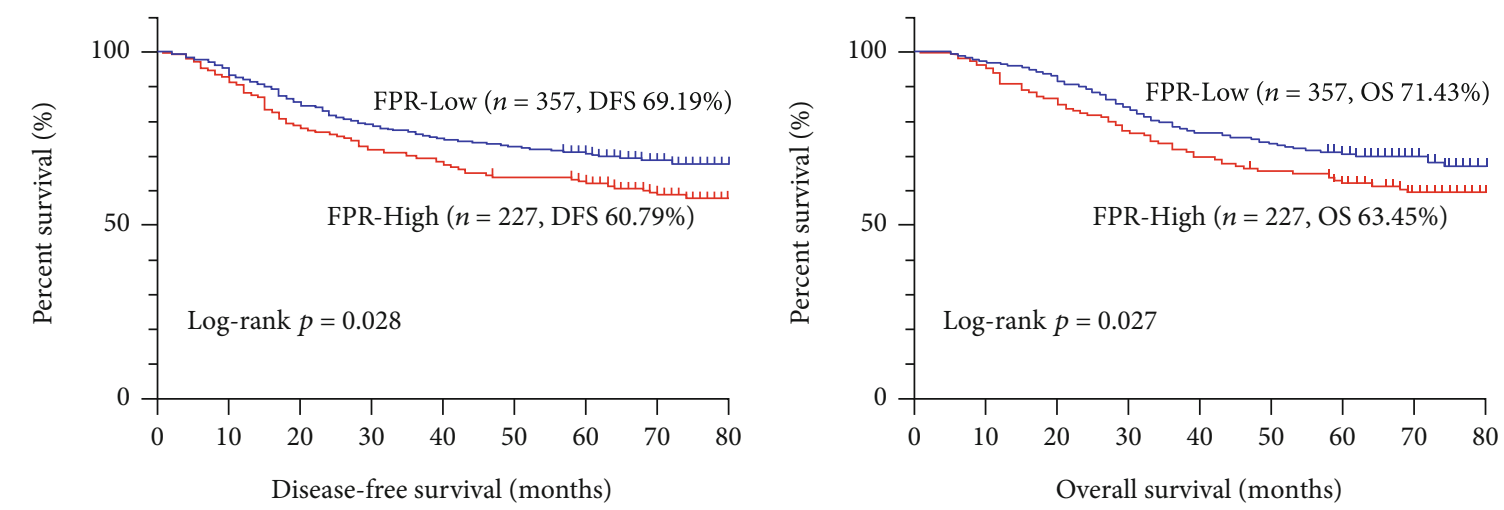

\begin{tabular}{cccccccccc}
\multicolumn{2}{l}{ Number at risk } \\
Low & 357 & 343 & 307 & 284 & 268 & 260 & 243 & 121 & 7 \\
High & 227 & 212 & 179 & 165 & 155 & 145 & 136 & 80 & 2
\end{tabular}

(a) \begin{tabular}{cccccccccc}
\multicolumn{2}{l}{ Number at risk } \\
Low & 357 & 349 & 338 & 303 & 278 & 271 & 249 & 125 & 7 \\
High & 227 & 219 & 190 & 176 & 160 & 151 & 140 & 80 & 2
\end{tabular}

(b)

FIgURE 1: Kaplan-Meier curves of FPR in stage I-III CRC patients. Notes: (a) disease-free survival and (b) overall survival.

calibration curve. Furthermore, the prognostic efficacy of survival nomograms and TNM stage was compared using time-dependent receiver operating characteristic (ROC) curve. SPSS 24.0 (IBM Corporation, Armonk, NY, USA) was used for all statistical analyses, and a $p$ value $<0.05$ with bilateral probability was considered statistically significant.

\section{Results}

3.1. General Study Population Information. CRC patient characteristics included in this study are summarized in Table 1. There were 363 males (62.2\%) and 221 females (37.8\%) with a median age of 59 years (range 17-92 years). Postoperative pathological results showed that the majority of patients had deep invasion (T3-4; 71.9\%) and no lymph node metastasis (58.4\%). There were 125 (21.4\%) patients with stage I CRC, 215 with stage II (36.8\%), and 244 with stage III (41.8\%). Moreover, 215 patients (36.8\%) had high preoperative CEA levels, and a large proportion of patients received postoperative adjuvant chemotherapy (50.7\%). The median values for PNI, NLR, LMR, and PLR were 46.13, 2.14, 3.60, and 137.41, respectively, which were used as the thresholds. Using the OS values, X-tile software determined the optimal FPR threshold to be 23.1. Based on this threshold value, 357 patients had a low FPR and 227 patients had a high FPR.

The correlation between FPR and clinicopathological factors is analyzed in Table 1. FPR was significantly positively correlated with age $(p=0.002)$, tumor size $(p=0.002)$, CEA $(p=0.002)$, fibrinogen $(p=0.002)$, PNI $(p<0.001)$, NLR $(p<0.001)$, and PLR $(p<0.001)$. It was also significantly inversely correlated with BMI $(p<0.001)$, albumin $(p<0.001)$, prealbumin $(p<0.001)$, and LMR $(p<0.001)$. In addition, patients with colon and vascular invasion were more likely to have high FPR.

3.2. Correlation of FPR with Postoperative Complications. To accurately evaluate the correlation between FPR and postoperative complications, $\geq$ grade II complications that required intervention were evaluated. Patients with a high FPR were more likely to have postoperative complications than those with a low FPR (16.3\% vs. 9.0\%, $p<0.007)$. To further determine the risk factors affecting postoperative complications, logistic regression analysis was performed based on various clinicopathologic factors. Age, hypertension, FPR, and blood loss were related to postoperative complications based on univariate analysis. However, multivariate analysis revealed that only high FPR (odd ratio $(\mathrm{OR})=1.785$, 95\% confidence interval $(\mathrm{CI})=1.062-2.999, p=0.024)$ and blood loss $(\geq 100 \mathrm{~mL} ; \quad \mathrm{OR}=1.956,95 \% \mathrm{CI}=1.151-3.326, p=0.013)$ were independent risk factors for postoperative complications (Table 2).

3.3. Comparison of Survival Curves between Low and High FPR. The median follow-up period was 65 months (range 1-80 months). A total of 185 patients (31.68\%) died, and $140(23.97 \%)$ had confirmed recurrence or distant metastasis upon the last follow-up. The survival curve between low and high FPR was compared using the Kaplan-Meier method with log-rank test. The results revealed that patients with a high FPR had a significantly worse DFS compared with patients with a low FPR $(p=0.028)$. Their corresponding five-year survival rates were $60.79 \%$ and $69.19 \%$ (Figure 1(a)). Similarly, patients with a high FPR had a dramatically worse OS compared with patients with a low FPR $(p=0.027)$ with corresponding five-year survival rates of $63.45 \%$ and $71.43 \%$, respectively (Figure $1(\mathrm{~b})$ ). The survival curves were further compared in each pathological stage. In stage I (Figures 2(a) and 2(d)), patients with a high FPR had a considerably worse DFS (70.21 vs. $87.18 \%, p=0.015$ ) and OS $(72.34 \%$ vs. $88.46 \%, p=0.017)$ than patients with a low FPR. In stages II (Figures 2(b) and 2(e)) and III (Figures 2(c) and 2(f)), the survival of patients with a high FPR was worse than that of patients with a low FPR, although these results were not statistically significant.

3.4. Correlation of FPR with Patient Survival. A variety of clinicopathological factors were incorporated into the Cox proportional hazards model for survival analysis. The 


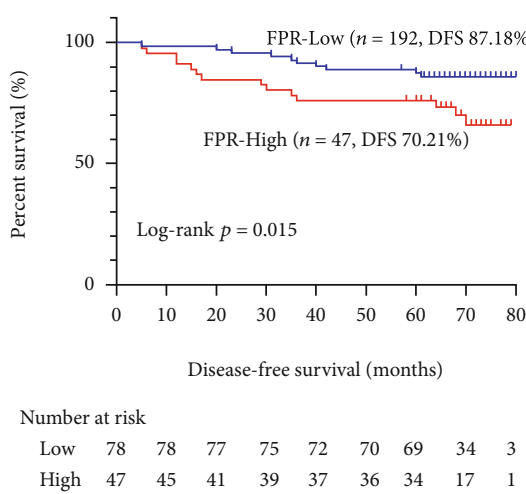

(a)

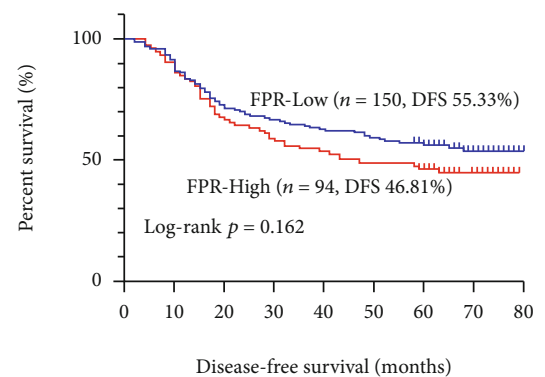

Number at risk $\begin{array}{llllllllll}\text { Low } & 150 & 138 & 110 & 101 & 95 & 90 & 84 & 44 & 3\end{array}$ $\begin{array}{llllllllll}\text { High } & 94 & 85 & 63 & 55 & 50 & 47 & 41 & 28 & 3\end{array}$

(c)

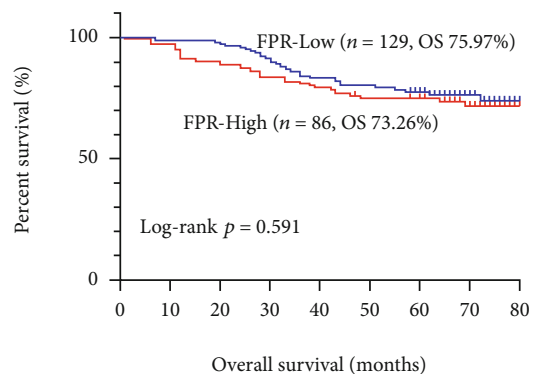

Number at risk

$\begin{array}{llllllllll}\text { Low } & 129 & 129 & 127 & 118 & 107 & 103 & 91 & 43 & 1\end{array}$ $\begin{array}{llllllllll}\text { High } & 86 & 84 & 78 & 73 & 70 & 65 & 62 & 35 & 2\end{array}$

(e)

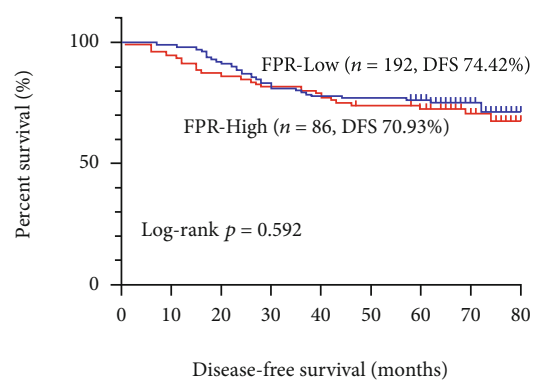

Number at risk

$\begin{array}{llllllllll}\text { Low } & 129 & 128 & 120 & 108 & 101 & 100 & 90 & 43 & 1\end{array}$

$\begin{array}{lllllllllll}\text { High } & 86 & 83 & 76 & 72 & 69 & 64 & 61 & 35 & 2\end{array}$

(b)

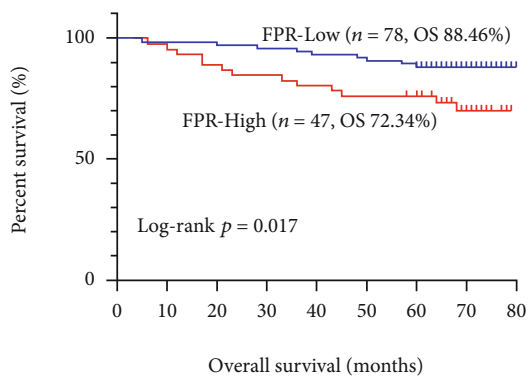

Number at risk

$\begin{array}{llllllllll}\text { Low } & 78 & 78 & 77 & 76 & 74 & 72 & 70 & 35 & 3\end{array}$

$\begin{array}{llllllllll}\text { High } & 47 & 46 & 42 & 40 & 38 & 36 & 34 & 17 & 1\end{array}$

(d)

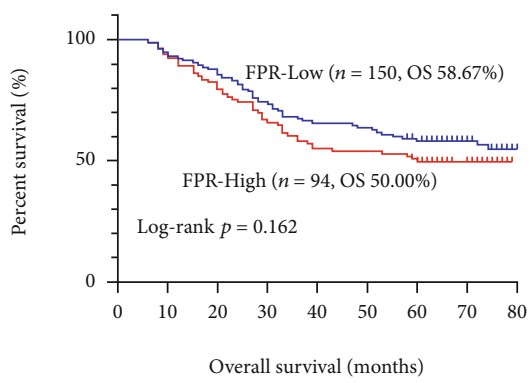

Number at risk

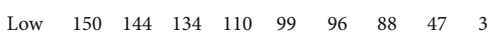

$\begin{array}{llllllllll}\text { High } & 94 & 89 & 78 & 63 & 52 & 51 & 44 & 28 & 3\end{array}$

(f)

FIGURE 2: Stratified Kaplan-Meier curves analysis of FPR based on TNM stage. Notes: (a) disease-free survival of FPR in stage I CRC patients; (b) disease-free survival of FPR in stage II CRC patients; (c) disease-free survival of FPR in stage III CRC patients; (d) overall survival of FPR in stage I CRC patients; (e) overall survival of FPR in stage II CRC patients; (f) overall survival of FPR in stage III CRC patients.

univariate analysis showed that the patients with deep invasion, lymph node metastasis, perineural invasion, vascular invasion, low histological grade, high CEA, advanced ASA grade, and high FPR had adverse DFS. However, in the multivariable analysis, only lymph node status (N1 vs. N0, hazard ratio $(\mathrm{HR})=1.378,95 \% \mathrm{CI}=0.958-1.983$; N2 vs. N0, HR $=3.922, \quad 95 \% \quad \mathrm{CI}=2.590-5.938 ; \quad p<0.001), \quad \mathrm{CEA}$ $(\mathrm{HR}=1.507,95 \% \mathrm{CI}=1.133-2.004, p=0.005)$, and $\mathrm{FPR}$ $(\mathrm{HR}=1.459,95 \% \mathrm{CI}=1.074-1.954, p=0.011)$ were the independent prognostic factors of DFS (Table 3). Similarly, age, depth of invasion, lymph node status, vascular invasion, histological grade, CEA, ASA grade, and FPR were statistically associated with OS in univariate analysis. However, in multivariate analysis, only lymph node metastasis (N1 vs. $\mathrm{N} 0, \mathrm{HR}=1.434,95 \% \mathrm{CI}=0.978-2.103$; N2 vs. N0, HR = 4.309, 95\% CI $=2.797-6.638 ; p<0.001)$, high $\mathrm{CEA}$ $(\mathrm{HR}=1.400,95 \% \mathrm{CI}=1.038-1.890, p=0.028)$, and high FPR $(\mathrm{HR}=1.405,95 \% \mathrm{CI}=1.034-1.909, p=0.038)$ were independent risk factors for OS (Table 4).

3.5. Establishing Prognostic Nomograms. Two simple and efficient prognostic nomograms were established for DFS (Figure 3(a)) and OS (Figure 3(b)) to predict the survival of stage I-III CRC patients undergoing surgical resection. The prognostic nomograms incorporated independent prognostic factors identified by multivariate analysis. The 
TABLE 3: The correlations between the disease-free survival and various clinicopathological factors.

\begin{tabular}{|c|c|c|c|c|}
\hline \multirow{3}{*}{ Variable } & \multicolumn{4}{|c|}{ Disease-free survival } \\
\hline & \multicolumn{2}{|c|}{ Univariate } & \multicolumn{2}{|c|}{ Multivariate } \\
\hline & HR (95\% CI) & $p$ value & HR $(95 \%$ CI $)$ & $p$ value \\
\hline Gender (female) & $0.873(0.652-1.168)$ & 0.360 & & \\
\hline Age (years) $(\geq 60)$ & $1.255(0.949-1.659)$ & 0.111 & & \\
\hline BMI & & 0.736 & & \\
\hline Low & 1 & & & \\
\hline Normal & $1.120(0.742-1.689)$ & & & \\
\hline High & $0.999(0.629-1.587)$ & & & \\
\hline Diabetes mellitus (positive) & $1.132(0.631-2.029)$ & 0.678 & & \\
\hline Hypertension (positive) & $1.229(0.860-1.756)$ & 0.258 & & \\
\hline Cardiovascular disease (positive) & $1.242(0.461-3.342)$ & 0.668 & & \\
\hline Depth of invasion (T3-4) & $1.951(1.365-2.789)$ & $<0.001$ & $1.320(0.907-1.922)$ & 0.147 \\
\hline Lymph node status & & $<0.001$ & & $<0.001$ \\
\hline N0 & 1.000 & & 1.000 & \\
\hline N1 & $1.661(1.190-2.320)$ & & $1.378(0.958-1.983)$ & \\
\hline N2 & $4.771(3.399-6.697)$ & & $3.922(2.590-5.938)$ & \\
\hline Tumor location (colon) & $0.852(0.643-1.129)$ & 0.266 & & \\
\hline Perineural invasion (positive) & $1.688(1.109-2.567)$ & 0.014 & $1.192(0.748-1.899)$ & 0.460 \\
\hline Vascular invasion (positive) & $1.882(1.338-2.646)$ & $<0.001$ & $1.188(0.805-1.753)$ & 0.385 \\
\hline Pathological type & & 0.335 & & \\
\hline Protrude type & 1.000 & & & \\
\hline Infiltrating type & $1.285(0.760-2.175)$ & & & \\
\hline Ulcerative type & $1.330(0.911-1.943)$ & & & \\
\hline Histological grade (low) & $1.799(1.226-2.640)$ & 0.003 & $1.310(0.883-1.943)$ & 0.179 \\
\hline Maximum tumor size $(\geq 5)$ & $1.234(0.934-1.630)$ & 0.140 & & \\
\hline CEA (high) & $1.738(1.315-2.296)$ & $<0.001$ & $1.507(1.133-2.004)$ & 0.005 \\
\hline ASA grade (III-IV) & $1.919(1.449-2.542)$ & $<0.001$ & $1.139(0.822-1.578)$ & 0.435 \\
\hline Type of surgery (open) & $0.906(0.686-1.197)$ & 0.486 & & \\
\hline Postoperative chemotherapy (yes) & $1.019(0.772-1.345)$ & 0.896 & & \\
\hline Albumin (low) & $1.112(0.781-1.584)$ & 0.555 & & \\
\hline Prealbumin (low) & $1.051(0.745-1.481)$ & 0.778 & & \\
\hline Fibrinogen (high) & $1.163(0.841-1.610)$ & 0.362 & & \\
\hline FPR (high) & $1.365(1.032-1.806)$ & 0.029 & $1.459(1.074-1.954)$ & 0.011 \\
\hline PNI (low) & $1.003(0.760-1.325)$ & 0.982 & & \\
\hline NLR (high) & $1.061(0.803-1.401)$ & 0.677 & & \\
\hline LMR (high) & $0.869(0.658-1.148)$ & 0.324 & & \\
\hline PLR (high) & $1.003(0.760-1.325)$ & 0.982 & & \\
\hline Blood loss $(\geq 100 \mathrm{~mL})$ & $1.110(0.840-1.466)$ & 0.464 & & \\
\hline Operation time ( $\geq 209 \mathrm{~min})$ & $1.224(0.926-1.618)$ & 0.155 & & \\
\hline
\end{tabular}

1-5-year DFS and OS in CRC patients were estimated by calculating the sum of the scores for each factor. The $C$ -indices of the prognostic nomograms for DFS and OS were $0.678(0.641-0.715)$ and $0.689(0.650-0.728)$, respectively. In addition, the calibration plot for DFS (Figures 4(a) and 4(b)) and OS (Figures 4(c) and 4(d)) at postoperative three- and five-year follow-up demonstrated an optimal agreement between the predicted prognostic nomograms and actual observations. The TNM staging system is widely recognized as the most effective scoring system to evaluate the prognosis in CRC patients.
The predictive ability between the prognostic nomograms and TNM staging system was further compared using time-dependent ROC. The area under the ROC curve for the prognostic nomograms was larger than that for the TNM stage at the three-year survival point (Figures 5(a) and $5(\mathrm{c}))$ as follows: DFS $-0.700,95 \% \mathrm{CI}=0.651-0.750$; TNM stage $-0.656,95 \% \mathrm{CI}=0.611-0.702$ and OS, 0.729 , $95 \% \mathrm{CI}=0.678-0.780$; and $\mathrm{TNM}$ stage-0.674, $95 \% \mathrm{CI}=$ $0.627-0.720$. The area under the ROC curve for the prognostic nomograms was also larger than that for the TNM stage at the five-year survival point (Figures $5(\mathrm{~b})$ and $5(\mathrm{~d})$ ) 
TABLE 4: The correlations between the overall survival and various clinicopathological factors.

\begin{tabular}{|c|c|c|c|c|}
\hline \multirow{3}{*}{ Variable } & \multicolumn{4}{|c|}{ Overall survival } \\
\hline & \multicolumn{2}{|c|}{ Univariate } & \multicolumn{2}{|c|}{ Multivariate } \\
\hline & $\operatorname{HR}(95 \% \mathrm{CI})$ & $p$ value & HR (95\% CI) & $p$ value \\
\hline Gender (female) & $0.843(0.622-1.142)$ & 0.270 & & \\
\hline Age (years) $(\geq 60)$ & $1.407(1.051-1.883)$ & 0.022 & $1.529(1.122-2.086)$ & 0.007 \\
\hline BMI & & 0.765 & & \\
\hline Low & 1 & & & \\
\hline Normal & $1.001(0.629-1.592)$ & & & \\
\hline High & $1.004(0.717-1.408)$ & & & \\
\hline Diabetes mellitus (positive) & $1.115(0.571-2.179)$ & 0.750 & & \\
\hline Hypertension (positive) & $1.216(0.838-1.763)$ & 0.304 & & \\
\hline Cardiovascular disease (positive) & $1.590(0.508-4.975)$ & 0.426 & & \\
\hline Depth of invasion (T3-4) & $2.008(1.378-2.926)$ & $<0.001$ & $1.409(0.947-2.098)$ & 0.091 \\
\hline Lymph node status & & $<0.001$ & & $<0.001$ \\
\hline N0 & 1.000 & & 1.000 & \\
\hline N1 & $1.624(1.145-2.302)$ & & $1.434(0.978-2.103)$ & \\
\hline $\mathrm{N} 2$ & $4.805(3.389-6.813)$ & & $4.309(2.797-6.638)$ & \\
\hline Tumor location (colon) & $0.815(0.608-1.093)$ & 0.172 & & \\
\hline Perineural invasion (positive) & $1.487(0.944-2.343)$ & 0.087 & & \\
\hline Vascular invasion (positive) & $1.751(1.226-2.502)$ & 0.002 & $1.112(0.763-1.621)$ & 0.581 \\
\hline Pathological type & & 0.329 & & \\
\hline Protrude type & 1.000 & & & \\
\hline Infiltrating type & $1.413(0.826-2.418)$ & & & \\
\hline Ulcerative type & $1.325(0.891-1.971)$ & & & \\
\hline Histological grade (low) & $1.919(1.298-2.838)$ & 0.001 & $1.495(0.994-2.248)$ & 0.053 \\
\hline Maximum tumor size $(\geq 5)$ & $1.298(0.972-1.733)$ & 0.077 & & \\
\hline CEA (high) & $1.698(1.272-2.267)$ & $<0.001$ & $1.400(1.038-1.890)$ & 0.028 \\
\hline ASA grade (III-IV) & $1.990(1.485-2.666)$ & $<0.001$ & $1.104(0.783-1.558)$ & 0.572 \\
\hline Type of surgery (open) & $1.122(0.841-1.497)$ & 0.435 & & \\
\hline Postoperative chemotherapy (yes) & $1.003(0.752-1.339)$ & 0.981 & & \\
\hline Albumin (low) & $1.173(0.808-1.701)$ & 0.401 & & \\
\hline Prealbumin (low) & $1.067(0.747-1.524)$ & 0.723 & & \\
\hline Fibrinogen (high) & $1.233(0.883-1.720)$ & 0.219 & & \\
\hline FPR (high) & $1.383(1.035-1.848)$ & 0.028 & $1.405(1.034-1.909)$ & 0.030 \\
\hline PNI (low) & $1.048(0.785-1.398)$ & 0.751 & & \\
\hline NLR (high) & $1.031(0.773-1.376)$ & 0.835 & & \\
\hline LMR (high) & $0.891(0.668-1.189)$ & 0.434 & & \\
\hline PLR (high) & $1.001(0.750-1.334)$ & 0.999 & & \\
\hline Blood loss $(\geq 100 \mathrm{~mL})$ & $1.155(0.865-1.542)$ & 0.327 & & \\
\hline Operation time ( $\geq 209 \mathrm{~min})$ & $1.286(0.962-1.718)$ & 0.090 & & \\
\hline
\end{tabular}

as follows: $\mathrm{DFS}-0.710,95 \% \mathrm{CI}=0.664-0.756$ and $\mathrm{TNM}$ stage $-0.657,95 \% \mathrm{CI}=0.613-0.701 ;$ OS $-0.718,95 \% \mathrm{CI}=$ $0.671-0.765$ and TNM stage, $0.649,95 \% \mathrm{CI}=0.604-0.694$.

\section{Discussion}

Systemic inflammation is an important hallmark of CRC [4]. Systemic inflammation can change tumor microenvironment, thereby promoting tumorigenesis and increasing proliferation, migration, and tumor cell immune escape $[6,24]$. Systemic inflammation is usually determined using biochem- ical blood routine indicators, which have been reported in many studies to be associated with CRC progression and prognosis [25-27]. Nutritional status is another important factor affecting cancer patient prognosis. A gradual nutritional status decline can suppress tumor immunity, leading to tumor spread and disease progression [28, 29]. Our previous studies have found that perioperative malnutrition may increase the incidence of postoperative complications and delay the recovery of postoperative function, leading to prolonged hospitalization, reduced life treatment, and even poor survival outcomes $[26,30]$. Various prognostic indicators 


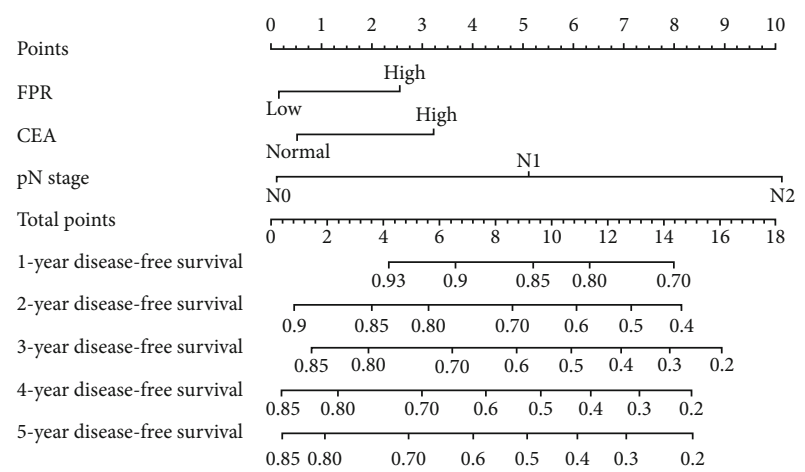

(a)
Points

FPR

CEA

pN stage

Age (years

Total Points

1 -year overall survival

2-year overall survival

3-year overall survival

4-year overall survival

5 -year overall survival

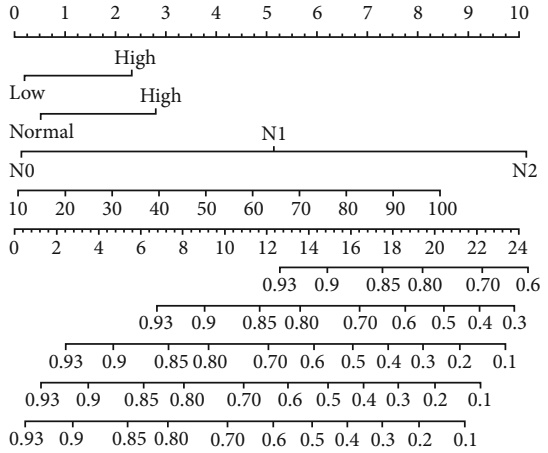

(b)

FIGURE 3: Construction of prognostic nomograms with FPR in stage I-III CRC patients for predicting 1-5-year DFS (a) and OS (b) in stage IIII CRC patients.

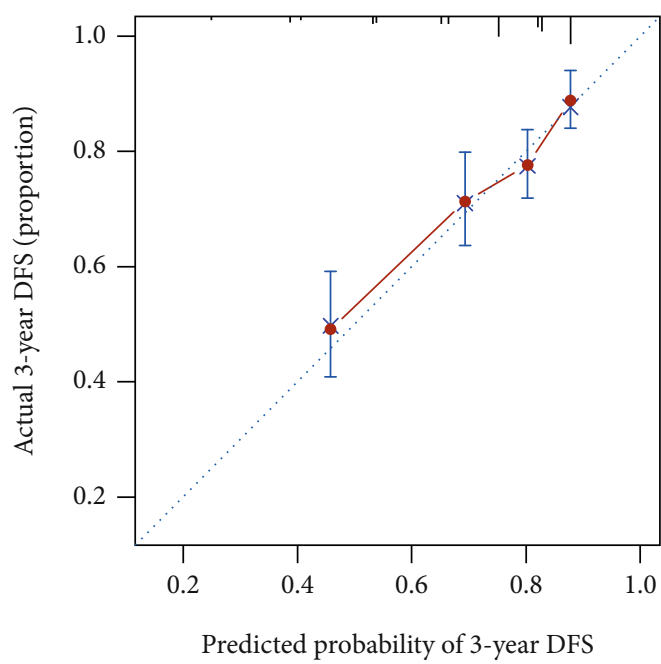

(a)

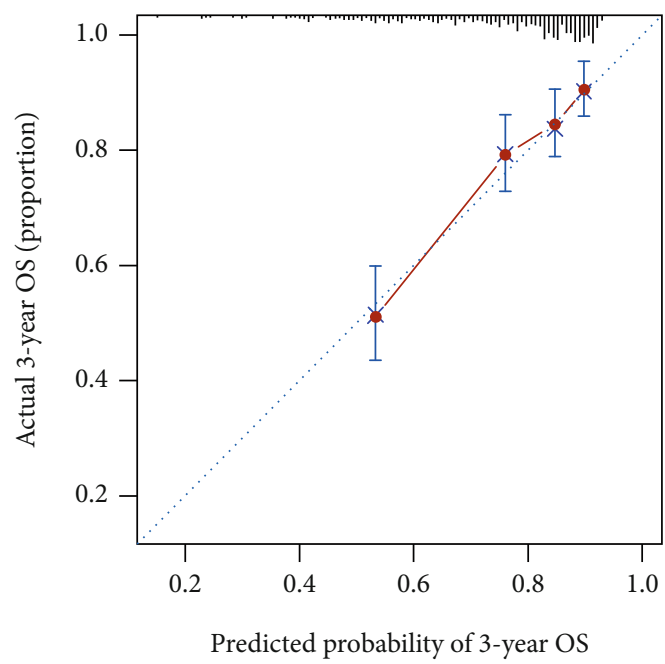

(c)

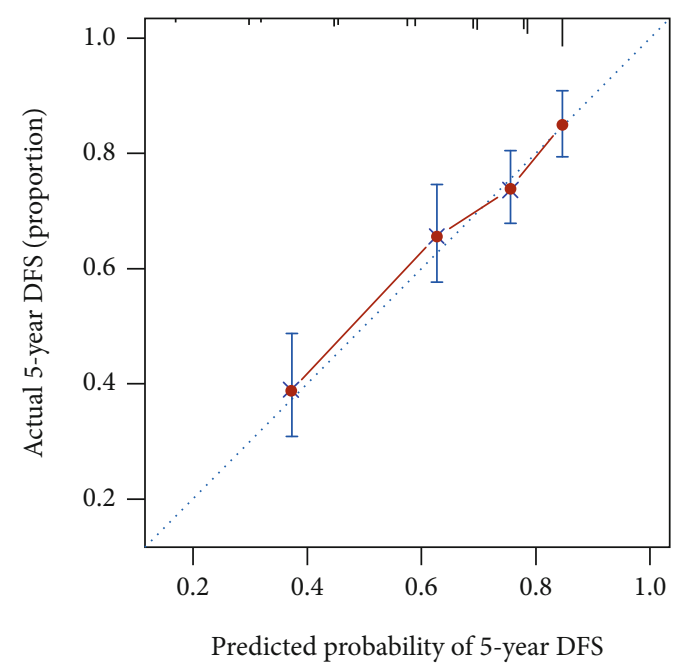

(b)

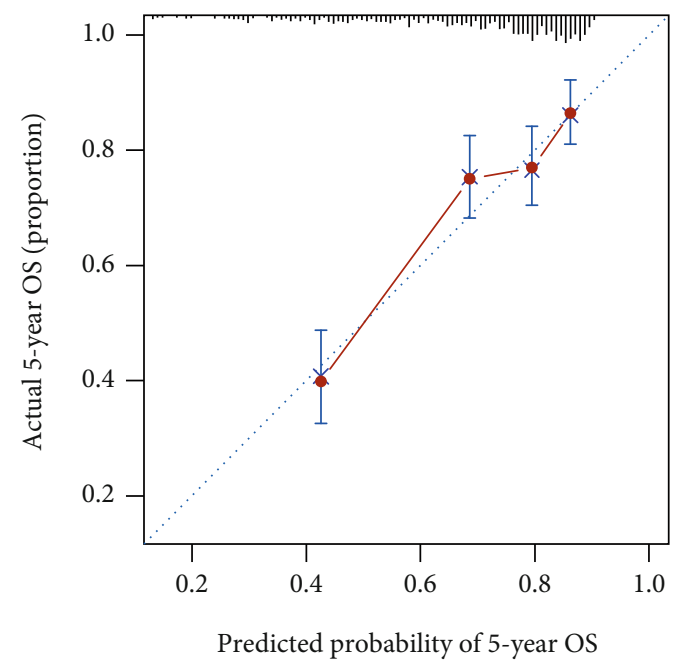

(d)

FIgURE 4: The calibration curves for predicting 3-year DFS (a), 5-year DFS (b), 3-year OS (c), and 5-year OS (d) in stage I-III CRC patients. 


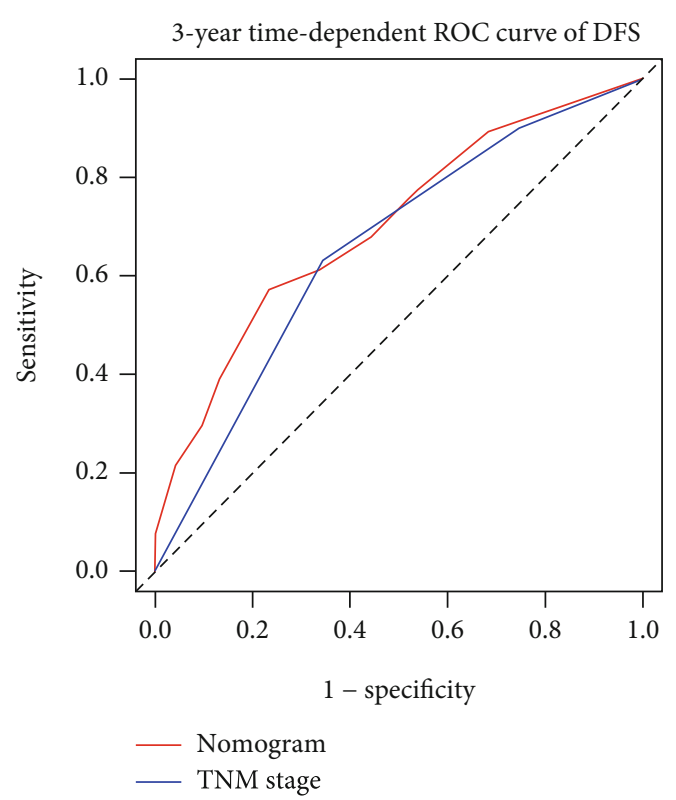

(a)

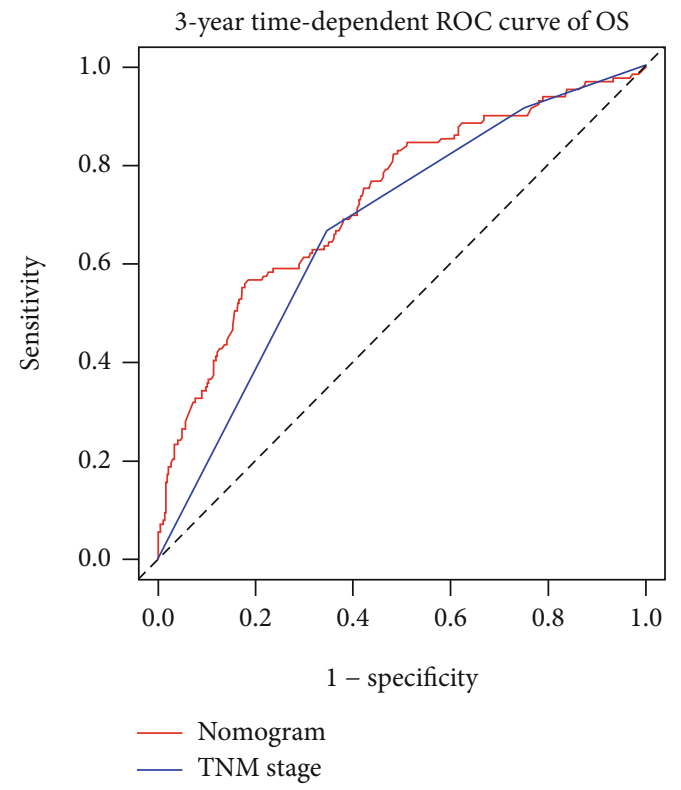

(c)

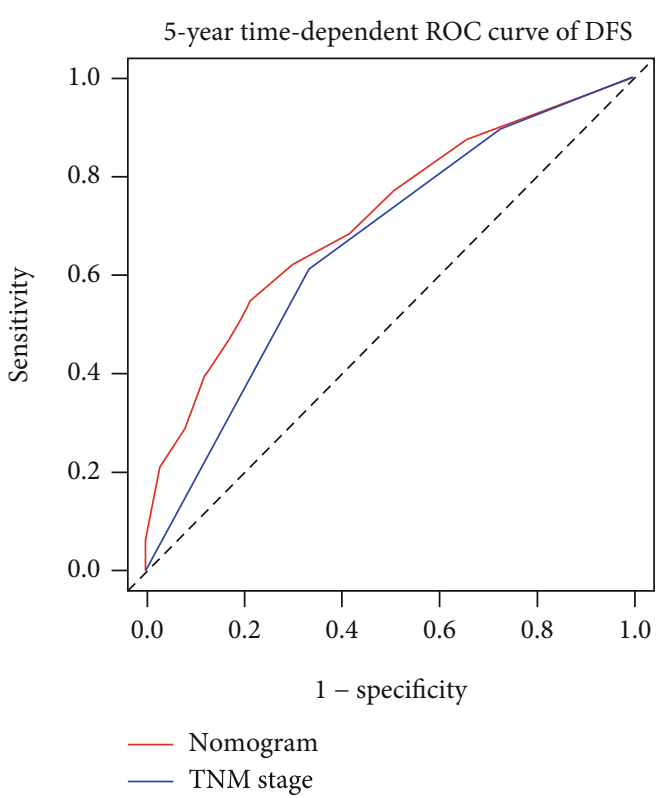

(b)

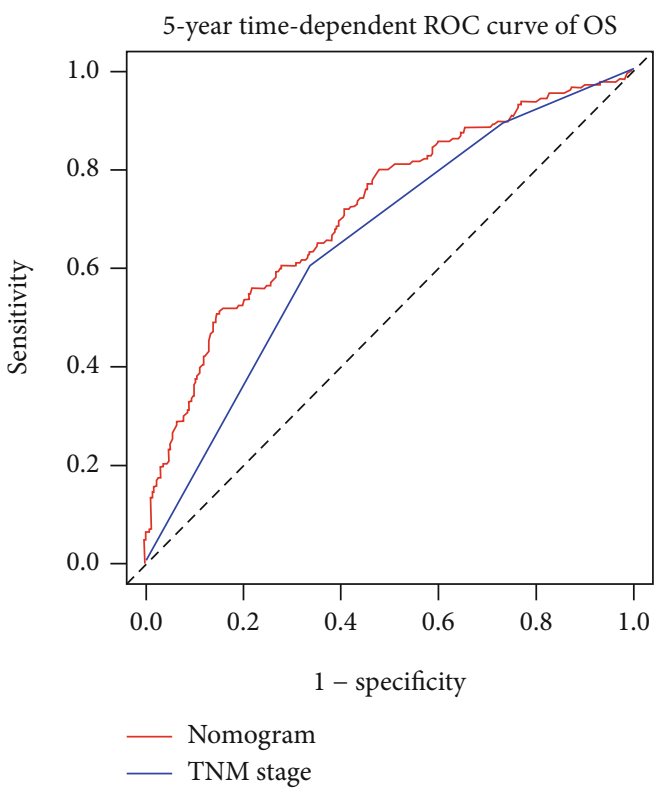

(d)

FIGURE 5: Comparison of the ability of prognostic nomograms and TNM stage for predicting prognosis in stage I-III CRC patients at 3-year and 5-year point. Notes: (a) DFS at 3-year point; (b) DFS at 5-year point; (c) OS at 3-year point; (d) OS at 5-year point.

based on systemic inflammatory and nutritional status have shown good performance in predicting the clinical outcomes in CRC patients, including Glasgow outcome score [31], modified Glasgow outcome score [32], and C-reactive protein/albumin [33].

The present study investigated the correlation between FPR and clinicopathological factors, complications, and survival in stage I-III CRC patients undergoing surgical resection. Correlation analysis results showed that high FPR was associated with low BMI, advanced age, and colon tumors, indicating that an increase in FPR may reflect the poor physical condition and nutritional status of patients. In addition, FPR is also correlated with tumor size and vascular invasion, suggesting that this biological indicator may be related to the aggressive cancer phenotype, which is consistent with previous studies $[9,34]$. The relationship between FPR and complications was also assessed for the first time, revealing that patients with a high FPR had a higher incidence of complications, while high FPR was an independent risk factor for complications. In addition, survival analysis confirmed that FPR is an independent prognostic factor affecting both DFS and OS in CRC patients. In the stratified survival curve analysis, the survival rate for the low FPR group was significantly higher than that for the high FPR group in stage I. There were no statistical differences in stage II and III CRC, suggesting that FPR may be used for prognosis assessment in early stage 
CRC patients. This result may be explained by a greater influence of inflammatory nutrition-related factors on early tumor development compared to other factors. Tumor invasion and metastasis gradually acquire a dominant role as the tumor progresses, while the impact of inflammatory nutrition-related factors is weakened. Two prognostic nomograms were constructed to individually predict clinical outcomes, and both the $C$-index and calibration plot confirmed that these nomograms were a reliable risk stratification tool. The nomograms combine the pathological characteristics of the tumor itself, inflammatory nutritional biomarkers, and tumor markers and can provide a personalized prognostic risk assessment for each patient. The ability of these nomograms to predict CRC patient prognosis was compared to the TNM stage. The nomograms were superior to the TNM stage in predicting three- and five-year survival in CRC patients, which showed that they have certain advantages in individually predicting CRC patient prognosis and can be used to develop personalized postoperative followup and treatment strategies for CRC patients.

The reasons why FPR is related to CRC patient prognosis remain unclear. However, there are several possible mechanisms. Fibrinogen is a potential factor affecting the activity and biological behavior of tumor cells [15]. Palumbo et al. have found that lymphatic metastasis is reduced in mice with fibrinogen deficiency and suggested that fibrinogen is an important factor that maintains continuous adhesion and survival of tumor cells in the vasculature of target organs and promotes metastasis [35]. Zheng et al. have reported that fibrinogen and platelets promote mutual aggregation around tumor cells to form a platelet-fibrinogen protective film, thereby protecting tumor cells from natural killer cells [36]. Prealbumin is not only a commonly used nutritional marker but also an acute phase negative protein, which is negatively correlated with inflammation. Zhou et al. have reported that prealbumin, which has a shorter half-life, can be affected by acute changes in protein balance earlier and respond to nutritional needs faster than albumin, making it an effective predictor of short-term efficacy after gastrectomy [37]. Shen et al. have found that prealbumin can be used in combination with lymphocytes to predict long-term prognosis of gastric cancer [38]. Albumin is another important indicator for the assessment of nutrition and inflammation. However, albumin, prealbumin, and fibrinogen alone were not factors affecting postoperative complications and long-term prognosis of CRC patients in the present study. Because FPR balances the effects of inflammation and nutrition, it can more comprehensively reflect the biological status of patients. Patients with a high FPR represent an upregulation of fibrinogen and a decrease in prealbumin, which indicate that patients have a poor nutritional status and cancer-related inflammation burden. Chen et al. have suggested that high FPR represents significant severe chronic inflammation and malnutrition that increase chemotherapy resistance, while circulating FPR can help predict the progression and survival of patients with left-sided metastatic CRC [39]. Sun et al. have reported that FPR combined with tumor markers can assist in the identification of patients requiring adjuvant chemotherapy and help predict CRC patient prognosis [40].
The present study was the first to investigate the prognostic value of FPR in stage I-III CRC patients undergoing surgical resection. The results confirmed that FPR upregulation is related to the invasion biology and malnutrition of CRC patients. They also demonstrated that FPR is an independent predictor of short- and long-term prognosis in CRC patients. Notably, this study has some limitations. This was a singlecenter retrospective study with potential bias in patient selection and medical record inaccuracy. In addition, although the prognostic nomograms have proven to be superior to TNM staging in the present study, further large-scale studies from multiple centers are needed to verify these results in the future.

\section{Conclusions}

The present study confirmed that FPR is a potential indicator for predicting short- and long-term prognosis in stage I-III CRC patients undergoing surgical resection.

\section{Data Availability}

The datasets generated and analyzed during the current study are not publicly available, because the data came from the hospital's independent and closed digital medical record management system. But they are available from the corresponding author on reasonable request.

\section{Conflicts of Interest}

The authors declare that they have no conflicts of interest.

\section{Acknowledgments}

This work was supported by the 2019 Innovation Project of Guangxi Graduate Education (JGY2019052).

\section{References}

[1] F. I. Bray, J. Ferlay, I. Soerjomataram, R. L. Siegel, L. A. Torre, and A. Jemal, "Global cancer statistics 2018: GLOBOCAN estimates of incidence and mortality worldwide for 36 cancers in 185 countries," CA: A Cancer Journal for Clinicians, vol. 68, no. 6, pp. 394-424, 2018.

[2] D. I. Obrand and P. H. Gordon, "Incidence and patterns of recurrence following curative resection for colorectal carcinoma," Diseases of the Colon and Rectum, vol. 40, no. 1, pp. 15-24, 1997.

[3] H. L. Rao, J. W. Chen, M. Li et al., "Increased intratumoral neutrophil in colorectal carcinomas correlates closely with malignant phenotype and predicts patients' adverse prognosis," PLoS One, vol. 7, no. 1, article e30806, 2012.

[4] J. Terzić, S. Grivennikov, E. Karin, and M. Karin, "Inflammation and colon cancer," Gastroenterology, vol. 138, no. 6, pp. 2101-2114.e5, 2010.

[5] Z. Mei, Y. Liu, C. Liu et al., "Tumour-infiltrating inflammation and prognosis in colorectal cancer: systematic review and meta-analysis," British Journal of Cancer, vol. 110, no. 6, pp. 1595-1605, 2014. 
[6] A. Mantovani, P. Allavena, A. Sica, and F. Balkwill, "Cancerrelated inflammation," Nature, vol. 454, no. 7203, pp. 436444, 2008.

[7] S. Friis, A. H. Riis, R. Erichsen, J. A. Baron, and H. T. Sørensen, "Low-dose aspirin or nonsteroidal anti-inflammatory drug use and colorectal cancer risk: a population-based, case-control study," Annals of Internal Medicine, vol. 163, no. 5, pp. 347$355,2015$.

[8] D. Gupta and C. G. Lis, "Pretreatment serum albumin as a predictor of cancer survival: a systematic review of the epidemiological literature," Nutrition Journal, vol. 9, no. 1, p. 69, 2010.

[9] L. Huang, Z. Mo, Z. Hu et al., "Diagnostic value of fibrinogen to prealbumin ratio and gamma-glutamyl transpeptidase to platelet ratio in the progression of AFP-negative hepatocellular carcinoma," Cancer Cell International, vol. 20, no. 1, p. 77, 2020.

[10] J. Zhang, S. Q. Li, Z. H. Liao et al., "Prognostic value of a novel FPR biomarker in patients with surgical stage II and III gastric cancer," Oncotarget, vol. 8, no. 43, pp. 75195-75205, 2017.

[11] J. F. Feng, L. Wang, Y. H. Jiang, and X. Yang, “A novel prognostic index in patients with resectable esophageal squamous cell carcinoma: fibrinogen/prealbumin ratio," Revista de Investigacionclinica, vol. 72, no. 1, pp. 46-54, 2020.

[12] S. Tang, L. Lin, J. Cheng et al., "The prognostic value of preoperative fibrinogen-to-prealbumin ratio and a novel FFC score in patients with resectable gastric cancer," BMC Cancer, vol. 20, no. 1, p. 382, 2020.

[13] D. W. Sun, L. An, and G. Y. Lv, "Albumin-fibrinogen ratio and fibrinogen-prealbumin ratio as promising prognostic markers for cancers: an updated meta-analysis," World Journal of Surgical Oncology, vol. 18, no. 1, p. 9, 2020.

[14] A. Sahni and C. W. Francis, "Vascular endothelial growth factor binds to fibrinogen and fibrin and stimulates endothelial cell proliferation," Blood, vol. 96, no. 12, pp. 3772-3778, 2000.

[15] P. J. Simpson-Haidaris and B. Rybarczyk, "Tumors and fibrinogen. The role of fibrinogen as an extracellular matrix protein," Annals of the New York Academy of Sciences, vol. 936, pp. 406-425, 2001.

[16] K. Lu, Y. Zhu, L. Sheng, L. Liu, L. Shen, and Q. Wei, "Serum fibrinogen level predicts the therapeutic response and prognosis in patients with locally advanced rectal cancer," HepatoGastroenterology, vol. 58, no. 110-111, pp. 1507-1510, 2011.

[17] Y. Wang, W. Yin, Z. Wang et al., "Pretreatment plasma fibrinogen as an independent prognostic indicator of prostate cancer patients treated with androgen deprivation therapy," Prostate Cancer and Prostatic Diseases, vol. 19, no. 2, pp. 209-215, 2016.

[18] J. Obata, N. Tanaka, R. Mizuno et al., "Plasma fibrinogen level: an independent prognostic factor for disease-free survival and cancer-specific survival in patients with localised renal cell carcinoma," BJU International, vol. 118, no. 4, pp. 598-603, 2016.

[19] A. Shenkin, "Serum prealbumin: is it a marker of nutritional status or of risk of malnutrition?," Clinical Chemistry, vol. 52, no. 12, pp. 2177-2179, 2006.

[20] F. Yang, L. Wei, X. Huo, Y. Ding, X. Zhou, and D. Liu, "Effects of early postoperative enteral nutrition versus usual care on serum albumin, prealbumin, transferrin, time to first flatus and postoperative hospital stay for patients with colorectal cancer: a systematic review and meta-analysis," Contemporary Nurse, vol. 54, no. 6, pp. 561-577, 2018.

[21] W. Gu, G. Zhang, L. Sun et al., "Nutritional screening is strongly associated with overall survival in patients treated with targeted agents for metastatic renal cell carcinoma," Journal of Cachexia, Sarcopenia and Muscle, vol. 6, no. 3, pp. 222230, 2015.

[22] J. Huang, Y. Wang, Y. Yuan et al., "Preoperative serum prealbumin as an independent prognostic indicator in patients with localized upper tract urothelial carcinoma after radical nephroureterectomy," Oncotarget, vol. 8, no. 22, pp. 3677236779, 2017.

[23] D. Dindo, N. Demartines, and P. A. Clavien, "Classification of surgical complications: a new proposal with evaluation in a cohort of 6336 patients and results of a survey," Annals of Surgery, vol. 240, no. 2, pp. 205-213, 2004.

[24] C. Lucas, N. Barnich, and H. Nguyen, "Microbiota, inflammation and colorectal cancer," International Journal of Molecular Sciences, vol. 18, no. 6, article 1310, 2017.

[25] H. Kubo, Y. Murayama, T. Arita, Y. Kuriu, M. Nakanishi, and E. Otsuji, "The prognostic value of preoperative neutrophil-tolymphocyte ratio in colorectal cancer," World Journal of Surgery, vol. 40, no. 11, pp. 2796-2802, 2016.

[26] S. Tang, H. Xie, J. Kuang, F. Gao, J. Gan, and H. Ou, "The value of geriatric nutritional risk index in evaluating postoperative complication risk and long-term prognosis in elderly colorectal cancer patients," Cancer Management and Research, vol. 12, pp. 165-175, 2020.

[27] J. H. Chen, E. T. Zhai, Y. J. Yuan et al., "Systemic immuneinflammation index for predicting prognosis of colorectal cancer," World Journal of Gastroenterology, vol. 23, no. 34, pp. 6261-6272, 2017.

[28] K. Barao, M. A. V. Cavagnari, P. S. Fucuta, and N. M. Forones, "Association between nutrition status and survival in elderly patients with colorectal cancer," Nutrition in Clinical Practice, vol. 32, no. 5, pp. 658-663, 2017.

[29] K. Norman, C. Pichard, H. Lochs, and M. Pirlich, "Prognostic impact of disease-related malnutrition," Clinical Nutrition, vol. 27, no. 1, pp. 5-15, 2008.

[30] H. Xie, Y. Gong, J. Kuang et al., "Computed-tomographydetermined sarcopenia is a useful imaging biomarker for predicting postoperative outcomes in elderly colorectal cancer patients," Cancer Research and Treatment, vol. 52, no. 3, pp. 957-972, 2020.

[31] Y. Liu, X. He, J. Pan, S. Chen, and L. Wang, "Prognostic role of Glasgow prognostic score in patients with colorectal cancer: evidence from population studies," Scientific Reports, vol. 7, no. 1, p. 6144, 2017.

[32] J. H. Park, D. G. Watt, C. S. D. Roxburgh, P. G. Horgan, and D. C. McMillan, "Colorectal cancer, systemic inflammation, and outcome: staging the tumor and staging the host," Annals of Surgery, vol. 263, no. 2, pp. 326-336, 2016.

[33] Y. Fan, S. Xiang, Z. Dai, C. Zou, X. Wang, and Z. Gao, "Prognostic significance of $\mathrm{C}$-reactive protein to albumin ratio in colorectal cancer patients: a meta-analysis," International Journal of Colorectal Disease, vol. 34, no. 6, pp. 1105-1111, 2019.

[34] L. Zhang, Q.-G. Chen, S.-Q. Li et al., "Preoperative fibrinogen to prealbumin ratio as a novel predictor for clinical outcome of hepatocellular carcinoma," Future Oncology, vol. 15, no. 1, pp. 13-22, 2019.

[35] J. S. Palumbo, K. W. Kombrinck, A. F. Drew et al., "Fibrinogen is an important determinant of the metastatic potential of circulating tumor cells," Blood, vol. 96, no. 10, pp. 3302-3309, 2000. 
[36] S. Zheng, J. Shen, Y. Jiao et al., "Platelets and fibrinogen facilitate each other in protecting tumor cells from natural killer cytotoxicity," Cancer Science, vol. 100, no. 5, pp. 859-865, 2009.

[37] J. Zhou, N. Hiki, S. Mine et al., "Role of prealbumin as a powerful and simple index for predicting postoperative complications after gastric cancer surgery," Annals of Surgical Oncology, vol. 24, no. 2, pp. 510-517, 2017.

[38] Q. Shen, W. Liu, H. Quan et al., "Prealbumin and lymphocytebased prognostic score, a new tool for predicting long-term survival after curative resection of stage II/III gastric cancer," British Journal of Nutrition, vol. 120, no. 12, pp. 1359-1369, 2018.

[39] Q. G. Chen, L. Zhang, F. Sun et al., "Elevated FPR confers to radiochemoresistance and predicts clinical efficacy and outcome of metastatic colorectal cancer patients," Aging, vol. 11, no. 6, pp. 1716-1732, 2019.

[40] F. Sun, H. X. Peng, Q. F. Gao et al., "Preoperative circulating FPR and CCF score are promising biomarkers for predicting clinical outcome of stage II-III colorectal cancer patients," Cancer Management and Research, vol. 10, pp. 2151-2161, 2018. 